\title{
Transaction Costs can Encourage Coasean Bargaining
}

Accepted for publication in Public Choice

\author{
by Alex Robson
}

Department of Accounting, Finance and Economics,
Griffith Business School,
Nathan Campus, Nathan, Qld, 4111, AUSTRALIA
email: a.robson@ @riffith.edu.au
Ph: +61-7-3735-7644. Facsimile: +61-7-3735-3719

\begin{abstract}
When there are three parties, instability problems brought about by the emptiness of the core of the corresponding cooperative game may cause the Coase Theorem to fail, even when other more direct impediments to bargaining are low. We show that the standard Coasean bargaining game involving three parties is strategically equivalent to an asymmetric three-player majority game. Hence, when there are three parties, instability problems will cause the Coase Theorem to fail if and only if the core of the corresponding three-player majority game is empty. We use this equivalence result to derive all instances in which the Coase Theorem will and will not hold with three parties, and show that a priori, such instability problems are likely to be rare - the Coase Theorem will actually hold most (over $80 \%$ ) of the time. We also demonstrate that it is always possible to find a set of transaction costs which, when introduced into a frictionless bargaining situation, will cause an empty core to become non-empty. In other words, transaction costs can mitigate instability problems: situations exist in which the presence of transaction costs will cause the Coase Theorem to hold when, in the absence of those direct transaction costs, it would fail to hold. When there are three parties, rather than hindering agreements, the existence of direct transaction costs can sometimes - but not always - reduce instability and encourage Coasean bargaining.
\end{abstract}

Keywords: Coase Theorem, externalities, transaction costs, cooperative games JEL Classification Codes: C71, C78, D23, D62, K0 


\section{Introduction}

There are two versions of the Coase (1960) theorem that are commonly referred to in the literature. ${ }^{1}$ The efficiency version states that if transaction costs are sufficiently low, then the initial legal regime or assignment of property rights will not prevent the parties from reaching an agreement in which all possible gains from trade have been exploited. The invariance version states that if transaction costs are sufficiently low, then bargaining will always lead to the same level of the activity which generates the externality, irrespective of the initial legal regime or assignment of property rights. ${ }^{2}$

Coase's result has a number of important implications for public choice analysis and the role of government and other social institutions. If transaction costs are sufficiently low, there is no efficiency justification for government intervention in the form of Pigovian taxes or subsidies. Indeed, these interventions may serve only to inhibit economic efficiency. In addition, legal rules derived from case law will have no bearing on the efficiency of the final allocation of resources. As long as some set of legal rules is in place, parties can costlessly bargain around them until all gains from trade are exploited. Hence, with low transaction costs, institutions such as courts will be unable to affect the efficiency of the final allocation of resources. Other scholars have extended Coase's analysis to the political system. As Buchanan (1973) and more recently Wittman (1989, 1995), Parisi (2003) and Luppi and Parisi (2012) have argued, in a world of low transaction costs, exchanges of votes (i.e., logrolling) between minorities and the majority may result in efficient outcomes. On the other hand, if transaction costs are high, then very different conclusions may follow. ${ }^{3}$

Coase's original analysis examined a situation with two parties. In an important and insightful paper, Aivazian and Callen (1981) argued that when there are three parties,

\footnotetext{
${ }^{1}$ See Parisi (2008) for a recent summary of the literature on the Coase Theorem.

${ }^{2}$ Cooter and Ulen (2012), Robson and Skaperdas (2008) and Robson (2012) distinguish between these two versions.

${ }^{3}$ In the "farmers' road building problem" studied by Tullock (1959) and Buchanan and Tullock (1962), logrolling under majority rule produces an inefficiently high level of government spending. Coase (1981) states that "We do not do well to devote ourselves to a detailed study of the world of zero transaction costs, like augurs divining the future by minute inspection of the entrails of a goose."
} 
both versions of the Coase Theorem can fail to hold. This can happen because of the empty core problem, wherein any agreement between all three parties is unstable. It is possible that agreements between two parties which exclude the third are so profitable that the individual opportunity cost of each of those two parties entering an agreement with the third party may exceed the benefits. Hence any agreement between all three parties will be susceptible to coalitions of two players breaking away, and both versions of the Coase Theorem can fail to hold.

Following Coase's (1981) suggestion that the empty core problem disappears when there are transaction costs, Bernholz (1997) argues that the empty core problem can be solved by the appropriate use of penalty clauses which increase the costs of breaking agreements. ${ }^{4}$ Aivazian and Callen (2003), Parisi (2003) and Luppi and Parisi (2012) show that stable outcomes for three-party bargaining problems are possible when coalition agreements are deemed binding and enforceable, so that instability does not persist. On the other hand, Aivazian and Callen (2003) also developed a numerical counterexample to Coase's (1981) general claim.

In light of Aivazian and Callen's analysis, it is useful to distinguish, as they do, between two conceptually different types of impediments that parties may face in trying to reach an agreement. ${ }^{5}$ First, there are the costs of physically arranging negotiations, signing an agreement, and so on. For the purposes of this paper and for the sake of precision and clarity, we will refer to these costs as "direct transaction costs". These kinds of costs may be present even when there are only two parties involved. Second, bargaining impediments may arise because of coalitional instability. These may arise even if it is costless physically to arrange negotiations and sign an agreement. For the purposes of this paper, we refer to these as "instability costs". These kinds of impediments may be present only when three or more parties participate.

\footnotetext{
${ }^{4}$ These kinds of costs are important, but are not the focus of the present analysis. Instead, we focus on the costs of making agreements.

5 Aivazian and Callen (2003: 288) state that "it is important to differentiate between transaction costs (when the core exists) and costs due to the empty core because each has different implications for rationalizing institutional arrangements."
} 
Coase (1988) explains that the assumption of no transaction costs in his original analysis should be interpreted as including both types of impediments. On this interpretation, instability costs are just another aspect of transaction costs which the theorem assumes away. ${ }^{6}$ However, rather than grouping both types of costs together, this paper examines them separately and studies the interaction between them. Our analysis assesses the likelihood of coalitional instability, and whether the introduction of direct transaction costs into an otherwise unstable three-player situation will improve matters. In doing so, we make a number of contributions to the law and economics literature, as well as the public choice literature regarding legislative bargaining, logrolling, and the contractarian approach to political economy and constitutions (Brennan and Buchanan 1985). Firstly, we develop a method for deriving all possible examples in which the Coase Theorem fails or holds when there are three parties. Secondly, we use this method to demonstrate that a priori, instability is relatively unlikely, and so the Coase Theorem will hold most of the time when direct transaction costs are low. Finally, we demonstrate that the introduction of direct transaction costs can cause an empty core to become non-empty. In other words, when direct transaction costs are present, the Coase Theorem can hold in cases where, in the absence of those direct transaction costs, it would fail to hold because of instability problems. Rather than hindering agreements, the presence of direct transaction costs can sometimes - but not always - reduce instability problems and encourage Coasean bargaining.

\section{The basic setup}

\subsection{The Aivazian-Callen (1981) example}

To illustrate the main issues, consider the following example, which is well known and first considered by Aivazian and Callen (1981). ${ }^{7}$ There are two factories, 1 and 2 , and a group of residents, R. Both factories emit pollution and this reduces the wellbeing of the residents. In the absence of production by the firms, the residents are assumed to enjoy utility of 40 . If both firms produce, the residents' utility falls to 24 .

\footnotetext{
${ }^{6}$ In a recent volume edited by Posner and Parisi (2013) on the Coase Theorem, a section is devoted to this debate, which includes the papers by Dixit and Olson (2000) and Anderlini and Fello (2006).

${ }^{7}$ The example also studied by Benoit and Kornhauser (2002).
} 
Therefore, when both factories produce they jointly impose an external cost of 16 $(=40-24)$ on the residents. Suppose that if only factory 1 produces it imposes an external cost of 9 on the residents, whilst if only factory 2 produces it imposes an external cost of 4 . The marginal external cost to the residents is therefore not constant. Finally, assume that if the factories produce alone, they can earn profits of 3 and 8 respectively. If they merge and produce together, then they can jointly earn profits of 15. This reflects an assumption of economies of scale in production.

Note that in this example there is actually more than one class of externality at work. ${ }^{8}$ The first two externalities are negative, and are caused by the factories reducing the residents' wellbeing. The second externality is a positive externality - both factories producing in combination increases their joint profits. We shall see, however, that the mere existence of this positive externality is not sufficient to make the Coase Theorem fail - it is the size of the positive externality that matters.

The efficient outcome in this example is for both factories not to produce. To better see how the example works, we transform it into a cooperative game with transferable utility. Let the characteristic function of this game be $v$. Suppose, first, that the factories can produce as much as they wish (a rule of no liability). Denote the value of the grand coalition by $v_{N}$. Then $v_{N}=40$, the total utility available to the parties when the factories and the residents agree that the factories should cease production. Notation for other possible coalitions is defined in a similar fashion. Under the no liability rule, the characteristic function of this cooperative game is:

$$
v_{1}=3, v_{2}=8, v_{R}=24, v_{12}=15, v_{1 R}=31, v_{2 R}=36, v_{N}=40
$$

Note that there are no direct transaction costs in this situation. However, Aivazian and Callen (1981) show that the core of this game is empty - there is no efficient agreement between the parties that is stable against threats by groups of two players to reach an agreement on their own.

Now suppose that the factories must first obtain the residents' permission to produce. Again, let the characteristic function of this game be $v$, and denote the value of the

\footnotetext{
${ }^{8}$ Mueller (2003: 30 also makes this point.
} 
grand coalition by $v_{N}$. Then again we have $v_{N}=40$. The characteristic function in this situation is:

$$
v_{1}=0, v_{2}=0, v_{R}=40, v_{12}=0, v_{1 R}=40, v_{2 R}=40, v_{N}=40
$$

No payment exists that the factories could make to persuade the residents to let them produce. The core of this game is non-empty and has a very simple structure: it is simply the point $x_{1}=0, x_{2}=0, x_{R}=40$. Note, however, that even though direct transaction costs are not an issue, the outcome is not the same as that under the no liability rule. Both versions of the Coase Theorem therefore fail to hold in this example.

\subsection{Modifying the example}

Only a slight modification of the previous example is needed to show how the result changes. Consider the same example, but now suppose that if the factories merge, they can realize joint profits of 12 rather than 15 . This seems like a trivial modification - after all, our previous example featured economies of scale; this example simply makes those synergies slightly smaller. However, modifying the example in this way reduces the opportunity cost of firms 1 and 2 entering into agreement with the residents. This reduction in opportunity cost means that the firms require less compensation for entering such an agreement, which in turn means that the residents' net benefit will be higher than in the previous case.

This slight change now renders efficient agreement stable. The characteristic function is now:

$$
v_{1}=3, v_{2}=8, v_{R}=24, v_{12}=12, v_{1 R}=31, v_{2 R}=36, v_{N}=40
$$

It is straightforward to show that the core of this game is non-empty. For example, any set of agreements satisfying:

$$
x_{1}=4-\alpha, x_{2}=8+\alpha, x_{R}=28 \text {, }
$$

where $0<\alpha<1$, is feasible and will be stable against deviations by a single party, as well as any deviation by a subcoalition of two parties. Hence, the core is non-empty, and both versions of the Coase Theorem will hold. 


\section{Deriving all three-player examples}

The previous discussion showed a minor modification of the payoffs can yield a nonempty core. A natural question, then, is how frequently instability is likely to arise. To understand when the Coase Theorem will hold and when it will not when there are three parties and no direct transaction costs, we consider the following asymmetric three-player majority game. ${ }^{9}$ In this game there are again three players, factories 1,2 and $R$. When all players all act together, they can obtain a payoff of $\$ 1$. Any two of them acting together can obtain a payoff smaller than 1, with different two player coalitions possibly obtaining different amounts. The characteristic function of this cooperative game is:

$$
v_{S}=\left\{\begin{array}{cc}
0 & \text { if }|S|=1 \\
\theta_{12} & \text { if } S=\{1,2\} \\
\theta_{1 R} & \text { if } S=\{1, R\} \\
\theta_{2 R} & \text { if } S=\{2, R\} \\
1 & \text { if }|S|=3
\end{array}\right.
$$

where $\left\{\theta_{12}, \theta_{1 R}, \theta_{2 R}\right\} \in(0,1)^{3}$. Suppose that the payments $x_{1}, x_{2}, x_{R}$ are in the core of this game. Then these payments must be non-negative, and we must have $x_{1}+x_{2}+x_{R}=1$ and $x_{1}+x_{2} \geq \theta_{12}, x_{1}+x_{R} \geq \theta_{1 R}, x_{2}+x_{R} \geq \theta_{2 R}$. But if this second set of inequalities holds, they must also hold if we sum them together, so we must have:

$$
2\left(x_{1}+x_{2}+x_{R}\right) \geq \theta_{12}+\theta_{1 R}+\theta_{2 R}
$$

But since $x_{1}+x_{2}+x_{R}=1$, this implies that the core is non-empty if and only if $\theta_{12}+\theta_{1 R}+\theta_{2 R} \leq 2$.

To understand the economic structure of the Aivazian-Callen example and how it relates to this asymmetric majority game, consider the 0-1 normalization of a cooperative game, which works as follows. ${ }^{10}$ Suppose that we have cooperative game with a characteristic function $v_{S}$, and which has $v_{i} \neq 0$ for some $i$, and $v_{N} \neq 1$. We seek to transform the payoffs of the game such that it is strategically equivalent to the

\footnotetext{
${ }^{9}$ This game is a more general case the symmetric majority game, which is studied by, for example, Osborne and Rubinstein (1995: 259).

${ }^{10}$ See, for example, Ordeshook (1986: 323).
} 
original game, but which has $v_{i}=0$ for all $i$, and $v_{N}=1$. This can be done by adding (not necessarily positive) numbers $z_{i}$ to the payoff of each individual to give them $v_{i}=0$. In other words, set $z_{i}=-v_{i}$. This number must be added to every coalition of which $i$ is a member. In particular, the new value of the grand coalition must be:

$$
K=v_{N}+\sum_{i} z_{i}
$$

Note that in the context of Coasean bargaining, $K=v_{N}+\sum_{i} z_{i}$ has the interpretation of the total available gains from trade, relative to autarky. Now, divide the new value of every coalition by $K$. The resulting characteristic function is strategically equivalent to the original game, since each individual's payoff has been scaled up by $z_{i}$ and divided by a constant $K$. Moreover, we have $v_{i}=0$ for all $i$, and $v_{N}=1$. Thus we have shown:

Lemma: Assume that there are no direct transaction costs. Then:

(a) Every three-player game in which the Coase Theorem fails to hold is strategically equivalent to an asymmetric three-player majority game with an empty core.

(b) Every three-player game in which the Coase Theorem holds is strategically equivalent to an asymmetric three-player weighted majority game with a non-empty core.

Recall that a cooperative game is cohesive if $v_{N} \geq \sum_{k=1}^{K} v_{S_{k}}$ for all partitions $\left\{S_{1}, \ldots, S_{K}\right\}$ of $N$. To study the Coase Theorem, we want to focus on situations in which it is efficient for the grand coalition to form. Hence we restrict our attention to cohesive games. But the asymmetric majority game is cohesive; hence the Lemma provides us with an algorithm for constructing all three-player cohesive cooperative games in which the Coase Theorem will and will not hold.

Proposition 1: Assume that there are no direct transaction costs. Let $v_{1}, v_{2}$ and $v_{R}$ 
be any three positive numbers, and let $v_{N}>v_{1}+v_{2}+v_{R}$. Then there exist numbers $\bar{v}_{12}$, $\bar{v}_{1 R}$ and $\bar{v}_{2 R}$ with $v_{n}>\bar{v}_{i j}>v_{i}+v_{j}$ for $i, j=1,2, R, i \neq j$ so that:

(a) If $v_{12}+v_{1 R}+v_{2 R}>\bar{v}_{12}+\bar{v}_{1 R}+\bar{v}_{2 R}$, the Coase Theorem will not hold; and

(b) If $v_{12}+v_{1 R}+v_{2 R} \leq \bar{v}_{12}+\bar{v}_{1 R}+\bar{v}_{2 R}$, the Coase Theorem will hold.

Moreover, the resulting cooperative game is cohesive.

Proof: To prove the result, we use the $0-1$ normalization and the previous Lemma. Let $v_{1}, v_{2}$ and $v_{R}$ be any three positive numbers, let $v_{N}>v_{12}+v_{1 R}+v_{2 R}$, and let $K=v_{N}-\left[v_{1}+v_{2}+v_{R}\right]$ be the efficiency gains from full cooperation. Let $\theta_{12}, \theta_{1 R}, \theta_{2 R}$ be any three numbers on the interval $(0,1)$ with the property that $\theta_{12}+\theta_{1 R}+\theta_{2 R}=2$.

Define $\bar{v}_{12}, \bar{v}_{1 R}$ and $\bar{v}_{2 R}$ implicitly by:

$$
\begin{aligned}
& \frac{\bar{v}_{12}-v_{1}-v_{2}}{K}=\theta_{12} \\
& \frac{\bar{v}_{1 R}-v_{1}-v_{R}}{K}=\theta_{1 R}
\end{aligned}
$$

and

$$
\frac{\bar{v}_{2 R}-v_{2}-v_{R}}{K}=\theta_{2 R}
$$

Then, by construction, any game with payoffs such that $v_{12}+v_{1 R}+v_{2 R}>\bar{v}_{12}+\bar{v}_{1 R}+\bar{v}_{2 R}$ will be equivalent to an asymmetric majority game with an empty core, and the Coase Theorem will fail to hold. Similarly, any game with payoffs such that $v_{12}+v_{1 R}+v_{2 R} \leq \bar{v}_{12}+\bar{v}_{1 R}+\bar{v}_{2 R}$ will be equivalent to an asymmetric majority game with a non-empty core, and the Coase Theorem will hold.

Note, too, that as long as the resulting game is derived from the asymmetric majority game, it will be cohesive, even if $\theta_{12}+\theta_{1 R}+\theta_{2 R}>2$. For example, as long as $v_{12}$ is constructed according to the above procedure, we will have: 


$$
\begin{aligned}
& v_{12}+v_{R}=K \theta_{12}+v_{1}+v_{2}+v_{R} \\
& =\left\{v_{N}-\left[v_{1}+v_{2}+v_{R}\right]\right\} \theta_{12}+v_{1}+v_{2}+v_{R} \\
& =\theta_{12} v_{N}+\left(1-\theta_{12}\right)\left[v_{1}+v_{2}+v_{R}\right] \\
& <\theta_{12} v_{N}+\left(1-\theta_{12}\right) v_{N}=v_{N}
\end{aligned}
$$

The equivalence between the Aivazian-Callen example and the asymmetric majority game is not just a theoretical curiosum. It allows us to construct examples and counterexamples. More importantly, it allows us to investigate how frequently we should expect to see the Coase Theorem failing because of the problems of instability associated with the empty core, as well as the effects of transaction costs. These two issues are addressed in the remainder of the paper.

\section{How likely is it that the Coase theorem will fail due to instability problems?}

As discussed above, the equivalence between the three-player bargaining game and the asymmetric majority game allows us to make precise statements about how likely it is that the core will be empty and the Coase Theorem will fail owing to instability problems. Since the two classes of games are equivalent, the failure of the Coase Theorem will hold with the same frequency that the core of the corresponding asymmetric majority game is non-empty, which requires $\left(\theta_{12}, \theta_{1 R}, \theta_{2 R}\right) \in(0,1)^{3}$ and $\theta_{12}+\theta_{1 R}+\theta_{2 R} \leq 2$. A priori, without any other information regarding the likelihood that various combinations of payoffs will occur, any set of payoffs in this set is equally likely. We therefore can show:

Proposition 2: Assume that there are no direct transaction costs and that all possible payoffs are equally likely. Then the Coase Theorem will hold most of the time. More precisely, it will hold with probability $5 / 6$.

Proof: We need to find the probability that $\theta_{12}+\theta_{1 R}+\theta_{2 R} \leq 2$, given that the points $\left(\theta_{12}, \theta_{1 R}, \theta_{2 R}\right)$ are independently uniformly distributed on the open unit cube $(0,1)^{3}$. This probability is given by: 


$$
\begin{aligned}
& \operatorname{Pr}\left(\theta_{12}+\theta_{1 R}+\theta_{2 R}<2\right)=1-\operatorname{Pr}\left(\theta_{12}+\theta_{1 R}+\theta_{2 R} \geq 2\right) \\
& =1-\int_{0}^{1} \int_{1-\theta_{2 R}}^{1} \int_{2-\theta_{1 R}-\theta_{2 R}}^{1} d \theta_{12} d \theta_{1 R} d \theta_{2 R} \\
& =1-\int_{0}^{1} \int_{1-\theta_{2 R}}^{1} 1-\left(2-\theta_{1 R}-\theta_{2 R}\right) d \theta_{1 R} d \theta_{2 R} \\
& =1-\int_{0}^{1} \int_{1-\theta_{2 R}}^{1}\left(\theta_{1 R}+\theta_{2 R}-1\right) d \theta_{1 R} d \theta_{2 R} \\
& =1-\int_{0}^{1}\left[\frac{\theta_{1 R}^{2}}{2}+\theta_{2 R} \theta_{1 R}-\theta_{1 R}\right]_{1-\theta_{2 R}}^{1} d \theta_{2 R} \\
& =1-\left\{\int_{0}^{1}\left[\frac{1}{2}+\theta_{2 R}-1\right]-\left[\frac{\left(1-\theta_{2 R}\right)^{2}}{2}+\theta_{2 R}\left(1-\theta_{2 R}\right)-\left(1-\theta_{2 R}\right)\right] d \theta_{2 R}\right\} \\
& =1-\left\{\int_{0}^{1}\left[\theta_{2 R}-\frac{1}{2}-\frac{\left(1-\theta_{2 R}\right)^{2}}{2}-\left(\theta_{2 R}-1\right)\left(1-\theta_{2 R}\right)\right] d \theta_{2 R}\right\} \\
& =1-\int_{0}^{1}\left[\theta_{2 R}-\frac{1}{2}+\frac{1-2 \theta_{2 R}+\theta_{2 R}^{2}}{2}\right] d \theta_{2 R} \\
& =1-\int_{0}^{1}\left[\frac{\theta_{2 R}^{2}}{2}\right] d \theta_{2 R}=1-\left[\frac{\theta_{2 R}^{3}}{6}\right]_{0}^{1}=\frac{5}{6}
\end{aligned}
$$

The interpretation of this result is straightforward: whilst it is always possible to construct combinations of payoffs such as those obtained by Aivazian and Callen (1981) which result in an empty core, with three players and if all possible payoff combinations are $a$ priori equally likely, these empty-core combinations are relatively unlikely. In three-player games, the failure of the Coase Theorem because of coalitional instability and the emptiness of the core is the exception rather than the rule.

This result is, of course, contingent on the assumption that every combination of payoffs is a priori equally likely to occur. This assumption may not always be reasonable. On the contrary, there is no natural probability structure on this game, ${ }^{11}$ and additional information may be available regarding the structure of payoffs, which suggests that some combinations are more likely than others. For example, there may be good economic reasons to believe that the two factories are likely to enjoy

\footnotetext{
${ }^{11}$ I am grateful to a referee for pointing this out.
} 
economies of scale. In that case, a different probability distribution of payoffs would need to be assumed, which places more weight on those payoff combinations where economies of scale are present. Proposition 2 simply states that in the absence of any information about the structure of payoffs - where a non-informative, uniform prior distribution is appropriate - it is reasonable to expect that, ex ante, instability problems will be unlikely, and that both versions of the Coase Theorem will hold. Under alternative assumptions, instability may be more (or less) likely than suggested by Proposition 2 .

\section{Transaction costs}

One of the most important insights of Coase's (1960) analysis is that if direct transaction costs are sufficiently large, then the legal rule or structure of initial property rights may matter for efficiency. Hence, there will exist efficiency enhancing and efficiency maximizing rules.

Avazian and Callen (2003) use a reasonable functional form for coalition formation costs to argue, by way of a counterexample, that if the core is empty in the absence of coalition formation costs, then it is also empty in the presence of such costs. Specifically, they assume that direct transaction costs take the following functional form:

$$
C_{S}=\left\{\begin{array}{cc}
|S|^{k} & \text { if }|S|>1 \\
0 & \text { otherwise }
\end{array}\right.
$$

where $k>1$. Recall that Coase (1981) suggested that the empty core problem disappears when there are transaction costs. On the other hand, Aivaizian and Callen (2003) developed a counterexample to show that a reasonable transaction costs technology for which Coase's (1981) suggestion does not hold. Proposition 3 below, which uses the method developed in Proposition 1, is of interest because it sits somewhere in between these two results and shows that Coase's (1981) claim must be modified slightly. It demonstrates that, counterintuitively - and in contrast to the standard treatments of the Coase theorem - the introduction of direct transaction costs can sometimes - but not always - reduce other impediments to bargaining. 
Proposition 3: For every three-player bargaining game without direct transaction costs having an empty core, there exists a transformed bargaining game with a set of positive, non-decreasing direct transaction costs that possesses a non-empty core.

Proof: Let the original payoffs be $v_{1}, v_{2}, v_{R}, v_{12}, v_{1 R}, v_{2 R}$ and $v_{N}$, and suppose that the core is empty. Choose transaction costs in the following way. For the grand coalition, we want $C_{N}$ to be sufficiently small so that there are still positive gains from trade. Thus:

$v_{N}-\left(v_{1}+v_{2}+v_{R}\right)-C_{N}=K-C_{N}>0$.

On the other hand, we do not want $C_{N}$ to be so small that it is exceeded by the transaction costs of two-player coalitions. Hence we will also require:

$C_{N}>K\left[3 \max \left\{\theta_{12}, \theta_{1 R}, \theta_{1 R}\right\}-2\right]$, which implies that $\frac{C_{N}}{3}>K\left[\max \left\{\theta_{12}, \theta_{1 R}, \theta_{1 R}\right\}-\frac{2}{3}\right]$

Now if $\frac{v_{12}-\left(v_{1}+v_{2}\right)}{K-C_{N}} \leq \frac{2}{3}$, set $C_{12}=0$. If not, set $C_{12}$ so that $\frac{v_{12}-C_{12}-\left(v_{1}+v_{2}\right)}{K-C_{N}}=\frac{2}{3}$.

Repeat these steps for $v_{1 R}$ and $v_{2 R}$. Note that if $C_{12}>0$ we have:

$$
v_{12}-C_{12}-\left(v_{1}+v_{2}\right)=\frac{2}{3}\left(K-C_{N}\right)
$$

so that:

$$
C_{12}=\theta_{12} K-\frac{2}{3}\left(K-C_{N}\right)=\frac{2}{3} C_{N}+\left(\theta_{12}-\frac{2}{3}\right) K<\frac{2}{3} C_{N}+\frac{C_{N}}{3}=C_{N},
$$

where the last inequality follows from the choice of $C_{N}$ that satisfied $C_{N}>K\left[3 \max \left\{\theta_{12}, \theta_{1 R}, \theta_{2 R}\right\}-2\right]$. Note that this game is strategically equivalent to a cohesive symmetric majority game with $\theta=2 / 3$, the core of which is non-empty.

The source of the transaction costs in Proposition 3 is that negotiations between parties are costly, and that costs tend to rise as the number of parties increases. However, the form of the transaction costs technology differs in important ways from the technology examined by Aivazian and Callen (2003) [see equation (5) above]. The transaction costs in (5) increase with the sizes of coalitions, and rise relatively rapidly as the number of parties increases. In contrast, although the overall 
transaction costs explored in Proposition 3 also increase with the number of parties, the costs do not rise as rapidly as they do in (5) when going from two to three parties. Furthermore, in Proposition 3 it is also possible for the size of the transaction costs to be different for different two-party coalitions. That is, the size of the transaction costs can depend on the identity of the two parties involved in negotiations. For example, going back to the Aivazian and Callen (1981) example, Proposition 3 allows for (but does not impose) the very real possibility that the cost of one the factories and the group of residents reaching an agreement may exceed the cost of the two factories reaching an agreement. This contrasts with the assumption implicit in the technology in equation (5), which is that all two-player coalitions face the same transaction costs. These modifications of the transaction costs technology together alter the opportunity costs of groups of two players deviating from the grand coalition and forming their own agreement. As a result, sticking with the grand coalition can sometimes - but not always - become relatively more attractive.

This result shows that as long as the direct transaction costs associated with reaching an agreement between two parties are sufficiently high (relative to the direct transaction costs associated with agreement making between three parties), the existence of these direct transaction costs can sometimes - but not always - reduce the opportunity costs of the grand coalition reaching an efficient agreement, and a cooperative game having an empty core will become a game with a non-empty core. Although the empty core problem does not always disappear when transaction costs are present, neither is it the case that the empty core problem never disappears.

It is straightforward to apply this result to the Aivazian and Callen (1981) example with an empty core. In their example, $K=5$, and $\theta_{12}=\theta_{1 R}=\theta_{2 R}=0.8$. Hence, the proof of Proposition 3 suggests that we will need $C_{N}>K\left[3 \max \left\{\theta_{12}, \theta_{1 R}, \theta_{1 R}\right\}-2\right]=5 \times(2.4-2)=2$. So, choose $C_{N}=3$. Also, the proof of Proposition 3 suggests that we should set:

$$
C_{12}=\theta_{12} K-\frac{2}{3}\left(K-C_{N}\right)=\frac{2}{3} C_{N}+\left(\theta_{12}-\frac{2}{3}\right) K=2+\left(0.8-\frac{2}{3}\right) 5=2 \frac{2}{3}
$$

with $C_{12}=C_{1 R}=C_{2 R}$.

Then the characteristic function for the new game with direct transaction costs is: 


$$
v_{1}=3, v_{2}=8, v_{R}=24, v_{12}=12 \frac{1}{3}, v_{1 R}=28 \frac{1}{3}, v_{2 R}=33 \frac{1}{3}, v_{N}=37,
$$

which is cohesive and has a non-empty core. Hence, the introduction of direct transaction costs into the Aivazian and Callen (1981) example can make an empty core non-empty. On the other hand, Proposition 3 and the results of Aivazian and Callen (2003) show that it is not the case that the introduction of arbitrary transaction costs will always have this effect. If transaction costs take a different form than those in Proposition 3, then the core of the modified game may still be empty. The main implication of Proposition 3 is that the presence of one type of impediment to bargaining (direct transaction costs) can sometimes - but not always - mitigate instability problems (and, indeed, completely eliminate this type of impediment). Direct transaction costs can encourage Coasean bargaining.

\section{Conclusion}

Following Aiavazian and Callen (2003), this paper has distinguished between two types of impediments to bargaining: direct transaction costs and bargaining impediments that may arise due to coalitional instability. Our analysis and results suggest that the two kinds of impediments to bargaining may interact in interesting and important ways. We first demonstrated the equivalence between the three-party bargaining game studied by Aivazian and Callen (1981) and an asymmetric majority game to derive a number of results. When there are no direct transaction costs, and if every combination of payoffs is a priori equally likely to occur, we should expect that the instability identified by Aivazian and Callen will occur relatively infrequently. Indeed, if all payoff combinations are equally likely, then Coasean bargaining is five times more likely than not.

The second main point in this paper is that direct transaction costs can sometimes but not always - mitigate the problems posed by instability. Specifically, we have shown that the those cases where instability could arise due to the emptiness of the core, there exists a set of direct transaction costs that eliminates completely the empty core as a source of instability and a barrier to Coasean bargaining.

\section{Acknowledgements}

I am grateful to Arye Hillman, Geoffrey Brennan, Jonathan Pincus, Ian MacKenzie and participants at the 2012 Australasian Public Choice Meetings for comments. 


\section{References}

Aivazian,V. and Callen, J.(1981) The Coase theorem and the empty core. Journal of Law and Economics, 24(1): 175-181.

Aivazian, V. and Callen, J. (2003) The core, transaction costs, and the Coase theorem. Constitutional Political Economy, 14: 287-299.

Anderlini, L. and Felli, L. (2006) Transaction costs and the robustness of the Coase theorem. Economic Journal, 116(508): 223-245.

Benoit, J-P. and Kornhauser, L. (2002) "Game Theoretic Analysis of Legal Rules and Institutions, Chapter 60 in Aumann, R. and Hart, S. (eds) Handbook of Game Theory with Economic Applications, Volume 3, London: North Holland.

Bernholz, P. (1997) Property rights, contracts, cyclical social preferences, and the Coase theorem: a synthesis. European Journal of Political Economy, 13: 419-442.

Brennan, G. and Buchanan, J. (1985) The Reason of Rules: Constitutional Political Economy, Cambridge, UK: Cambridge University Press.

Buchanan, J. (1973) The Coase theorem and the theory of the state. Natural Resources Journal, 13: 579-594.

Buchanan, J. and Tullock, G. (1962) The Calculus of Consent, Ann Arbor: University of Michigan Press.

Coase, R. (1960) The problem of social cost. Journal of Law and Economics, 3: 1-44.

Coase, R. (1981) The Coase theorem and the empty core: comment," Journal of Law and Economics, 24(1): 183-187.

Coase, R. (1988) "Notes on the Problem of Social Cost," Chapter 5 in Coase, R. The Firm, the Market, and the Law, Chicago: University of Chicago Press.

Cooter, R. and Ulen, T. (2012) Law and Economics, $6^{\text {th }}$ edition, New York: Prentice Hall.

Dixit, A. and Olson, M. (2000) Does voluntary participation undermine the Coase theorem? Journal of Public Economics, 76: 309-335.

Luppi, B. and Parisi, F. (2012) "Politics With(out) Coase," International Review of Economics, 59: 175-187. 
Mueller, D. (2003) Public Choice III, New York: Cambridge University Press.

Ordeshook, P. (1986) Game Theory and Political Theory, Cambridge: Cambridge University Press.

Osborne, M. and Rubinstein, A. (1994) A Course in Game Theory, Cambridge, MA: MIT Press.

Parisi, F. (2008) "Coase Theorem" in Durlauf, S. and Blume, L. (eds) The New Palgrave Dictionary of Economics, London: Palgrave Macmillan.

Parisi, F. (2003) Political Coase theorem. Public Choice, 115 (1/2): 1-36.

Posner, R. and Parisi, F. (eds) (2013) The Coase Theorem, 2 Volumes, Economic Approaches to Law Series, Cheltenham: Edward Elgar.

Robson, A. and Skaperdas, S. (2008) Costly enforcement of property rights and the Coase theorem. Economic Theory, 36: 109-128.

Robson, A. (2012) Law and Markets, London: Palgrave Macmillan.

Tullock, G. (1959) Problems of majority voting. Journal of Political Economy, 67(6): 571-579.

Wittman, D. (1989) Why democracies produce efficient results. Journal of Political Economy, 97(6): 1395-1424.

Wittman, D. (1995) The Myth of Democratic Failure, Chicago: University of Chicago Press. 